\title{
Transposition
}

Musique et Sciences Sociales

\section{Emmanuel Brandl, Cécile Prévost-Thomas et Hyacinthe Ravet (ed.), 25 ans de sociologie de la musique en France, tome 2.}

Paris, L'Harmattan, 2012, 244 p.

\section{Annelies Fryberger}

\section{Q OpenEdition}

\section{Journals}

Édition électronique

URL : http://journals.openedition.org/transposition/152

DOI : 10.4000/transposition. 152

ISSN : 2110-6134

Éditeur

CRAL - Centre de recherche sur les arts et le langage

Référence électronique

Annelies Fryberger, «Emmanuel Brandl, Cécile Prévost-Thomas et Hyacinthe Ravet (ed.), 25 ans de sociologie de la musique en France, tome 2. ", Transposition [En ligne], 3 | 2013, mis en ligne le 01 mars 2013, consulté le 22 septembre 2020. URL : http://journals.openedition.org/transposition/152 ; DOI : https://doi.org/10.4000/transposition. 152

Ce document a été généré automatiquement le 22 septembre 2020

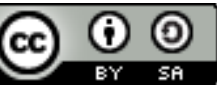

La revue Transposition est mise à disposition selon les termes de la Licence Creative Commons Attribution - Partage dans les Mêmes Conditions 4.0 International. 


\section{Emmanuel Brandl, Cécile Prévost- Thomas et Hyacinthe Ravet (ed.), 25 ans de sociologie de la musique en France, tome 2.}

Paris, L'Harmattan, 2012, 244 p. Annelies Fryberger

\section{RÉFÉRENCE}

Emmanuel Brandl, Cécile Prévost-Thomas et Hyacinthe Ravet (ed.), 25 ans de sociologie de la musique en France, tome 2, Paris, L'Harmattan, 2012, 244 p.

1 Notons d'emblée que la lecture de ce deuxième tome des 25 ans de la sociologie de la musique en France rend manifeste un fait notable concernant la sociologie de la musique : comme toute discipline, elle s'est construite et continue de se construire autour de figures tutélaires, auxquelles la plupart des auteurs de l'ouvrage se réfèrent. Il s'agit d'Howard S. Becker, Pierre Bourdieu et Pierre-Michel Menger, dont les réflexions ne cessent d'alimenter les différents articles de l'ouvrage. Becker en particulier semble avoir marqué profondément ce domaine : douze des quinze articles le citent, et au moins six auteurs utilisent sa conception des « mondes » musicaux pour structurer leur travail. L'ouvrage fondateur de Becker, Art Worlds, a été traduit et publié en France en $1988^{1}$, six ans après sa parution aux États-Unis. Depuis, il est un auteur incontournable de la sociologie de la musique en France. Son ouverture à la diversité musicale - qui transcende la distinction opérée traditionnellement entre le savant et le populaire -, ainsi que son plaidoyer pour un décloisonnement disciplinaire rencontrent une forte résonance dans le recueil : la sociologie de la musique en France puise ses outils et ses références dans des disciplines aussi diverses que la sociologie des professions et des sciences, l'ethnomusicologie, la musicologie, l'anthropologie, les 
études de genre, l'esthétique, ou la philosophie. C'est cette diversité qui fait sa force, et qui en même temps freine son institutionnalisation.

2 C'est d'ailleurs le problème que soulève Denis Laborde au sujet de l'ethnomusicologie. Il s'interroge, dans son article, sur la légitimité disciplinaire de l'ethnomusicologie, qui existe en France depuis plus longtemps que la sociologie de la musique et qui pourtant peine à se légitimer en tant que discipline. L'alternative centrale, à propos de laquelle il est difficile de trancher, est la suivante : la musique est-elle un fait social distinct, nécessitant des outils et des méthodes distincts et exigeant la formation de disciplines vouées à son étude uniquement? Ou bien est-elle le fruit d'une pratique créatrice qui serait mieux comprise en comparaison avec d'autres pratiques créatrices? Laborde analyse le désir de l'ethnomusicologie de faire de la musique un fait social distinct (ce qu'il qualifie d'" approche d'aval ») et, qui plus est, de distinguer entre tradition orale (le pré-carré de l'ethnomusicologie) et tradition savante (l'apanage de la musicologie). Mais une autre voie est possible (qu'il qualifie d' "approche d'amont») : si la musique est un fait social comme les autres, cette distinction a-t-elle vraiment un sens? Selon ce point de vue, la musique est à considérer comme le résultat de pratiques créatrices, et si notre but est d'étudier ces pratiques, la distinction faite entre musique populaire et musique savante, ou encore entre la musique et des objets issus d'autres pratiques créatrices, n'a plus de sens. En creux, on comprend que le désir de forger une discipline universitaire influe sur la façon dont les chercheurs abordent leur objet - une réflexion qui vaut d'ailleurs pour toute discipline.

3 Le débat concernant le savant et le populaire est repris par Romain Pudal dans sa contribution, où il est question du rôle et du pouvoir symbolique des détenteurs de la parole légitime (ici, les universitaires). Sa question principale, concernant le travail de Bourdieu, est la suivante : est-ce que celui-ci constate simplement un état de fait dans son travail sur la légitimité des pratiques culturelles, ou bien contribue-t-il par ce travail, justement, à créer des distinctions et de la légitimité ? Pudal souligne les dangers d'un travail sur l'art populaire; comme l'expérience de cet art est généralement muette, le chercheur s'empare du rôle de porte-parole, ce qui risque d'ôter ce droit de parole aux amateurs et de ne faire que renforcer le pouvoir symbolique des détenteurs de la parole légitime (p.237). Cela nous révèle à la fois l'intérêt et le danger d'une sociologie de la musique populaire: ce travail accorde, qu'on le veuille ou non, de la légitimité aux pratiques «illégitimes» et c'est le chercheur qui met en mots l'expérience de cette musique. C'est notre rôle dans ces mondes de musique, on pourrait dire, mais il faut être conscient des conséquences de ce travail, auxquelles on ne songe pas forcément.

4 Les travaux présentés dans ce tome montrent bien que les sociologues de la musique ont choisi, pour la plupart, "l'approche d'amont" explicitée par Laborde, où l'attention se concentre sur le faire, la musique étant considérée comme le résultat d'une pratique créatrice comparable à d'autres pratiques créatrices. Les pratiques musiciennes sont abordées directement dans la première partie de cet ouvrage, et l'on constate que la distinction entre musique savante et musique populaire n'a pas cours lorsqu'il est question de pratiques. On peut ainsi constater que les résultats auxquels aboutissent les recherches portant sur les pratiques de musiciens de tradition populaire peuvent alimenter le travail de ceux qui travaillent sur la musique savante, et vice versa. La contribution qui ouvre ce deuxième tome, celle de Pierre-Michel Menger, considère le monde de la musique dans sa totalité, sans distinction de genre. Il montre 
que l'on peut analyser les carrières de musiciens sans découper arbitrairement ce monde entre le savant et le populaire. Gildas de Séchelles s'inspire de l'approche de Menger pour établir sa distinction entre musiciens créateurs (ceux qui ont la maitrise du sens d'une activité musicienne) et musiciens interprètes (qui participent dans une moindre mesure à la production de sens). Son article prend pour objet les «musiques amplifiées ", mais ses conclusions sont d'une grande pertinence pour la compréhension de toute pratique créatrice.

5 Au fil de l'ouvrage, on observe que la distinction épistémologique entre le savant et le populaire n'est plus valable en sociologie de la musique et que l'amont et l'aval - le faire et l'œuvre - ne peuvent être analysés séparément. En gardant ces éléments essentiels à l'esprit, nous allons maintenant nous intéresser à certains argumentaires et approches marquants de ce riche volume.

6 S'inspirant de Foucault, Laborde constate que «ce qui est fait - de la musique s'explique par le faire et non pas le faire par ce qui est fait» (p. 207). Cela explique pourquoi plusieurs articles s'ouvrent sur la question : que fait-on quand on interprète / joue / fait / écoute de la musique? Maÿlis Dupont suit précisément cette approche : "que font les interprètes?» (p.131), se demande-t-elle en introduction et en conclusion de son texte. Dans le cas analysé, celui de la création d'une œuvre de musique contemporaine, ils sont successivement auteurs (dans leurs négociations avec la compositrice sur un texte inachevé), experts (capables de "produire sur demande [...] du matériau musical calibré », p. 131) et cobayes (observés en continu, sollicités pour expérimenter le dispositif d'enregistrement et les idées de la compositrice). Audelà de ces remarques sur le travail des interprètes, l'auteure remarque que la compositrice est souvent amenée à noter la performance des interprètes dans sa partition - c'est finalement leur performance de l'œuvre, négociée avec la compositrice, qui se trouve fixée dans la partition (p. 131). Ici, la division traditionnelle du travail se trouve bousculée: la distinction entre les rôles du compositeur et de l'interprète tend à se brouiller. Pour reprendre les termes de Gildas de Séchelles, le compositeur a un rôle plus important que les interprètes dans la production du sens de l'activité musicale, sans avoir le monopole de celle-ci.

7 La variabilité des rôles de l'interprète se trouve analysée sous un autre angle dans le travail de Gildas de Séchelles. Il fait une étude similaire à celle de Maÿlis Dupont, mais à visée plus générale, sur des musiciens de musiques amplifiées. Il étudie les différents rôles joués par les musiciens dans la production du sens et de l'identité d'un projet musical, et montre que les profits symboliques sont liés à l'implication dans cette production. Le «musicien créateur» (par opposition au "musicien interprète») conçoit et manipule le sens et le discours d'un projet musical ; il représente la figure de l'entrepreneur et agit comme employeur pour les autres intervenants. Dans les musiques amplifiées, les catégories de « compositeur » et d' «interprète » sont moins figées qu'en musique classique, et c'est peut-être pour cette raison qu'il est plus facile de voir que la distinction "créateur " / «interprète » est finalement une question de degré, et qu'un individu peut changer de rôles dans les projets divers dans lesquels il est engagé. Son investissement est plus grand quand son rôle est plus important dans la production du sens du projet (p. 71), et cet investissement peut se traduire en profits symboliques et économiques en cas de succès du projet. Mais pour que le succès soit au rendez-vous, «le travail de l'artiste consiste [...] à représenter le non-travail ou l'activité non contrainte» (p.74). Paradoxalement, pour convertir les profits 
symboliques en profits économiques, il faut participer à la production du sens d'un projet, sans avoir l'air (pour le public) de travailler. Il faut noter au passage que le regard du public serait également instrumental dans le processus de production de sens ; c'est un point que Franck Léard développe plus amplement dans son travail sur les musiciens de bar.

Un autre aspect à prendre en compte lorsque l'on analyse ce «faire » de la musique est la question de la communication: quand on travaille avec d'autres pour faire de la musique, ce qui n'est, on pourrait le postuler, jamais un travail exclusivement solitaire, comment communique-t-on? S'il faut parler de la musique, comment faire si tous les participants n'ont pas la même formation musicologique? Marc Perrenoud cherche la réponse à cette question après une enquête de terrain d'une dizaine d'années auprès de musiciens " ordinaires ». Son objet est constitué par les " musiques actuelles ", où « les répertoires cognitifs et discursifs mobilisés par les instrumentistes ordinaires dans leur pratique collective sont souvent très variés, très labiles » (p. 106). Cette déclaration laisse entendre qu'il existerait des genres où ces répertoires seraient plus uniformes (sans jamais l'être tout à fait), ce qui est effectivement le cas des étudiants de conservatoires en France, comme nous allons le voir plus en détails au sujet du travail de Pierre François. Perrenoud a fait un travail d'observation participante, et il fait état de trois modes de communication nécessaires pour "faire de la musique ensemble » (p.114) : analyse musicologique, vocalisation mimétique et description évocatrice. Ces différents modes sont utilisés en situation pour s'approprier le matériau musical intellectuellement et pratiquement. Cette étude analyse la manière dont les conventions sont créées et adaptées en situation et montre que la capacité à manier un vocabulaire musicologique est un signal de légitimité : "les "lettrés" du groupe se lançant des clins d'œil quand le leader s'empêtrait dans un lexique musicologique mal maîtrisé » (p. 110).

9 Mais le «faire» de la musique ne renvoie pas uniquement aux interactions entre musiciens. Quand on inclut le public dans son analyse, on tend à pratiquer une étude plus globale, celle qui appréhende les aspects commerciaux des mondes musicaux. Pierre-Michel Menger adopte cette perspective socio-économique dans ses nombreuses recherches dans ce domaine, et sa contribution à ce tome montre la fécondité de cette approche. Son analyse tente d'expliquer les fortes inégalités de gains que l'on constate dans les professions artistiques. En effet, la distribution des compétences et des qualifications ne correspond pas à la distribution des gains, et cette inégalité est particulièrement forte chez les musiciens. Plusieurs facteurs sont passés en revue pour expliquer ces inégalités : la relation entre formation et emploi, les écarts de talent, la consolidation de la réputation, la longévité dans la carrière, les tournois éliminatoires, la gestion du risque, la surestimation de soi et le caractère arbitraire du jugement. La clé de voûte de son analyse est le concept d' "incertitude »; c'est elle qui explique la rupture entre formation, compétences, qualifications et les gains que l'on peut espérer en retirer. Son hypothèse de base est que les critères de performance professionnelle ne sont pas clairement définis, ce qui fait que les résultats d'une activité créatrice ne peuvent pas être évalués en termes absolus (p.23). Il avance l'idée que les marqueurs divers de succès - consolidation de la réputation, réussite dans des tournois, etc. - ont pour fonction de réduire l'incertitude du jugement de valeur porté sur la qualité artistique d'un individu. Il n'évoque pas l'effet Matthieu dans son analyse, mais on peut le lire en filigrane : le succès appelle le succès, dans un monde où d'autres marqueurs de qualité sont plus difficiles à évaluer. Finalement, c'est la structure du marché - 
l'organisation par projets - qui structure le rapport au risque et à l'incertitude. Les acteurs sont obligés de faire l'arbitrage entre l'exploitation des compétences accumulées (certitude sur le résultat) et un comportement exploratoire (incertitude sur le résultat) (p. 33).

Pierre François rend hommage à son directeur de thèse, Pierre-Michel Menger, dans un article traitant du rôle des conservatoires dans l'insertion professionnelle des musiciens. Cette enquête, financée par la Direction de la musique, de la danse, des théâtres et des spectacles du Ministère de la Culture, a abouti à cette publication, entre autres. De ce texte riche en nuances, nous retenons l'analyse du rôle des diplômes dans le marché musical : ils n'agissent pas en tant que signaux comme sur d'autres marchés du travail fortement qualifiés (p.62), ce qui renforce l'hypothèse de Menger déjà discutée. François montre que ce que le conservatoire fournit d'essentiel serait plutôt les compétences techniques et un réseau professionnel. Après, ce sont les forces analysées par Menger qui entrent en jeu; le diplôme du conservatoire n'est pas un facteur explicatif fort du succès. Cette conclusion est à nuancer en fonction du type de carrière visée, surtout dans le cas du marché des solistes nationaux ou internationaux (p. 61).

11 Nous avons vu plus haut que le faire et l'œuvre sont tous les deux essentiels pour le travail d'un sociologue de la musique. Cependant, c'est le travail sur les œuvres musicales proprement dites qui se trouve parfois marginalisé dans les approches sociologiques. On observe un déplacement épistémologique dans la partie sur les œuvres musicales, ce qui peut se lire notamment dans le fait que T.W. Adorno ne soit cité que dans les articles présentés sous le titre « La question des œuvres musicales ». Si Becker est le père fondateur de la sociologie du «faire la musique », Adorno joue ce rôle pour la sociologie de l'œuvre musicale. La lecture de ce tome dans son ensemble nous montre que l'on peut croiser ces deux approches pour arriver à pratiquer une sociologie de la musique capable d'appréhender à la fois le faire et l'œuvre, sur un pied d'égalité.

12 Esteban Buch plaide pour une sociologie des œuvres «telles qu'écoutées par quelqu'un »; une sociologie des œuvres qui comprend donc la réception contextualisée de celles-ci. Il remarque qu'une analyse musicologique est nécessairement imprégnée de l'idéologie de la personne qui fait l'analyse, et il faut pouvoir en quelque sorte analyser l'analyse des œuvres en sociologie de la musique. Il nous donne un exemple de la façon dont on peut appliquer un tel programme à travers une étude de la polémique entre Hans Pfitzner et Alban Berg sur la manière d'analyser la Rêverie des Scènes d'enfant opus 15 de Robert Schumann. Avec cette étude de cas, il montre que «l'analyse musicale, autrement dit la description de la partition, [...] permet d'observer que les [approches de Pfitzner et de Berg] sont basées sur une perception correcte des traits objectifs, tout en privilégiant à chaque fois des phénomènes de saillance différents » (p.141). Cela montre clairement que nous ne pouvons pas faire abstraction de la personne qui écoute quand on pratique une sociologie des œuvres ${ }^{2}$ : l'écoute a des aspects sociaux et historiques qu'il ne faut pas ignorer.

13 Cette thèse est reprise par Jean-Marie Jacono, qui étudie le cas de Boris Goudonov. Il montre comment cette œuvre a évolué dans des contextes historiques et sociaux différents. Il postule que les « œuvres doivent être traitées comme des composantes actives de la vie en société, au même titre que d'autres facteurs" (p. 147). De cette façon, il choisit son camp parmi ceux qui ne conçoivent pas la musique comme un fait 
social distinct. L'exemple de l'opéra de Moussorgski, modifié plusieurs fois pendant le siècle qui a suivi sa composition, est révélateur: Rimski-Korsakov, notamment, l'a révisé « en fonction des attentes d'un groupe social qui va jouer un rôle actif dans sa transformation et sa reprise » (p. 150). C'est donc grâce à ces modifications que l'opéra a connu le succès qu'on lui connaît. En utilisant ce cas comme matière de base, il élabore une liste de critères qui doivent être pris en compte dans une sociologie de l'œuvre musicale inspirée, entre autres, par le travail de T.W. Adorno (p. 153).

L'article de Karim Hammou a dû être difficile à classer dans les parties présentées dans ce tome, puisque c'est le seul qui parvient à combiner sociologie du faire et sociologie de l'œuvre. C'est le seul qui traite activement à la fois de l'amont et de l'aval, et sa contribution fait l'effet d'une révélation des possibilités offertes par la sociologie de la musique. Son objet est très précis - l'usage du refrain dans les albums de rap français entre 1990 et 2004 - mais sa portée est grande. Il montre comment une multitude de facteurs, tels que les changements dans l'industrie du disque, la politique musicale du ministère de la culture, le rôle du DJ et du MC, ont concouru pour faire changer les conventions esthétiques du monde du rap en français. La figure du rappeur change au cours de cette période, grâce en partie à l'évolution de la musique assistée par ordinateur, et il passe de collaborateur à auteur dans ce système, en même temps que les DJs perdent ce statut pour jouer plutôt un rôle de médiateur. Ce changement du statut du DJ a un effet direct sur la forme des chansons de rap, et Hammou arrive à constater ce changement de statut en partant d'une analyse de l'œuvre : il constate que ce qu'il appelle le « refrain DJ » a cédé la place au « refrain versifié » pendant la période étudiée. Il apparaît clairement ici que les questionnements de nature esthétique, souvent abordés dans une analyse de l'œuvre, nécessitent un travail approfondi sur le faire. On en revient alors au constat foucaldien déjà cité : c'est le faire qui explique l'œuvre et non l'inverse (Laborde, p. 207). Hammou arrive à révéler les rapports de force qui ont abouti à ce changement de conventions artistiques et, ce faisant, démontre la fécondité d'une approche croisant une sociologie des conventions à la Becker et une sociologie de l'œuvre.

Il est difficile de rendre justice à l'ensemble des travaux présentés dans cet ouvrage très riche. Les préoccupations majeures qui en ressortent - la pertinence de la distinction populaire / savant et l'opposition faire / œuvre - sont traitées sous des angles divers, mais tous s'accordent pour dire que l'on perd du temps à vouloir à tout prix tracer une ligne étanche entre la musique savante et la musique populaire et que, même si l'on n'arrive pas à faire les deux soi-même tout le temps, l'entrecroisement d'une sociologie de l'œuvre et d'une sociologie du faire est riche en enseignements. Étant donnée la multitude d'approches possibles pour aborder la musique, on ne peut que conclure qu'elle est un fait social comme les autres, mais un fait social particulièrement fécond pour le sociologue. Nous pouvons apprendre beaucoup de choses en étudiant la musique. 


\section{NOTES}

1. BECKER, Howard Saul, Les Mondes de l'art, Paris, Flammarion, 1988.

2. La contribution de Michel Blanc à ce tome en serait un exemple. Ses préoccupations personnelles informent ouvertement son analyse de l'émergence du genre de l'opérette française. On ne peut qu'apprécier sa franchise, puisque les goûts du chercheur sont rarement présentés explicitement dans le travail sociologique sur la musique.

\section{AUTEURS}

\section{ANNELIES FRYBERGER}

Annelies Fryberger est doctorante contractuelle en première année à l'EHESS, sous la codirection de Nicolas Donin (Ircam) et Esteban Buch (EHESS/CRAL). Elle mène des recherches comparatives (France/États-Unis) sur les structures de financement en musique contemporaine et leur impact sur le processus créateur des compositeurs. Elle a obtenu son diplôme de master en 2012 de l'EHESS pour son travail sur la collaboration entre commanditaires et compositeurs en musique contemporaine. Américaine d'origine, elle a fait des études de français et de piano classique à Oberlin College et Conservatoire aux États-Unis. 